\title{
1 Sox8 is sufficient to reprogram ectoderm into ear vesicles and associated neurons
}

$6{ }^{1}$ Centre for Craniofacial and Regenerative Biology, King's College London, London SE1 9RT, UK

$7 \quad 2$ The Francis Crick Institute, London NW1 1AT, UK

8

$9 \$$ These authors contributed equally.

$10{ }^{*}$ Corresponding author: andrea.streit@kcl.ac.uk

$11{ }^{3}$ current address: Maijing Gene Diagnostics, Guangzhou 510030, China 
13 The vertebrate inner ear arises from a pool of progenitors with the potential to give rise to all

14 the sense organs and cranial ganglia of the head ${ }^{1-6}$. Here we explore the molecular

15 mechanisms that control ear specification from these precursors. Using a multi-omics

16 approach combined with loss-of-function experiments we identify a core transcriptional circuit

17 that imparts ear identity, along with non-coding elements that integrate this information. This

18 analysis places the transcription factor Sox8 at the top of the ear determination network.

19 Introducing Sox8 into cranial ectoderm not only converts non-ear cells into ear progenitors,

20 but also activates the cellular programmes for ear morphogenesis and neurogenesis. Thus,

21 Sox8 emerges as a master regulator of ear identity and may be a key factor for sense organ

22 cell reprogramming. 
Master regulators have the unique ability to reprogram cells from other cell types. They activate a set of transcription factors that in turn control the expression of proteins required for cell-specific functions.

The classic example is the transcription factor MyoD, which can convert fibroblasts into myogenic cells ${ }^{7}$. From insects to vertebrates, the transcription factor Pax6 induces ectopic eyes when misexpressed in non-eye cells ${ }^{8}$, however master regulators for other sense organs have not yet been discovered. Here we set out to identify such factors for the organ of hearing and balance, the vertebrate inner ear.

During development, the inner ear arises from the posterior border of the cranial neural plate.

Here, precursors for neural, neural crest, ear and sensory ganglia are initially intermingled, but segregate over time ${ }^{9,10}$. FGF signalling from adjacent tissues induces otic-epibranchial progenitors (OEPs), which subsequently converge to distinct territories: the otic and epibranchial placodes ${ }^{11-15}$

(Fig. 1a, e). While epibranchial cells produce sensory ganglia, the otic placode invaginates to form a vesicle, which is then transformed into the inner ear, containing many specialised cell types and associated neurons. Using transcriptional profiling, we recently defined the first gene regulatory network for early ear development ${ }^{16}$. However, the key factors that impart ear character remain unknown as do the non-coding regions that integrate the process. Here we use single cell RNAsequencing (scRNAseq) and pseudo-time analysis to predict transcriptional changes as progenitors undergo ear commitment. Using epigenomic profiling we also determine ear enhancers and their upstream regulators. To ensure transparency and reproducibility, we have developed a fully integrated workflow of the data analysis in Nextflow (available at https://alexthiery.github.io/oticreprogramming/). Together with functional experiments, we identify a minimal transcriptional circuit that defines ear identity comprising Sox8, Pax2 and Lmx1a, with Sox8 at the top of the hierarchy. Introduction of Sox8 alone into cranial ectoderm is sufficient to trigger the ear programme, to initiate ear morphogenesis by forming ear vesicles and to activate neurogenesis. Thus, using a multi-omics approach we have uncovered Sox8 as a master regulator of ear fate, which may in the future allow reprograming of specific sensory cell types. 
Dynamic changes in gene expression characterise the transition from progenitor to otic

commitment

To unravel the genetic hierarchy that controls how otic and epibranchial cells diverge, we first characterised the transcriptional profile of cells committed to each fate ${ }^{2}$ (Fig1. a-d, Extended Data

Fig. 1a, b). To label each cell population we used two enhancers driving EGFP, the novel otic Lmx1aE1 enhancer (see below) and the epibranchial enhancer Sox3U3 ${ }^{17}$. Reporter constructs were electroporated into the OEP territory of somite stage (ss) 3-5 embryos. At ss18-21, EGFP ${ }^{+}$cells were isolated by fluorescence-activated cell sorting (FACS) and processed for RNA-seq. Differential expression analysis identifies 103 and 319 genes upregulated in Lmx1aE1-EGFP and Sox3U3-EGFP expressing cells, respectively (log2FC > 1.5; adjusted p-value $<0.05$; Fig. 1d; Extended Data Fig. 1C, $\mathrm{d}$ and Supplementary Table1). This allows us to define the transcriptional states of definitive otic and epibranchial cells. common progenitor population to definitive otic and epibranchial cells. Pax2 is expressed throughout

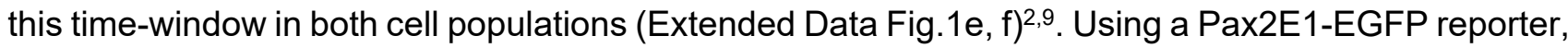
we isolated single cells by FACS from consecutive stages of ear specification: OEP (ss8-9), earlyplacode (ss11-12) and late-placode stage (ss14-15) and processed them for single-cell-RNA-seq (scRNAseq; Fig. 1e, f). To characterise cellular diversity, we first looked at groups of genes coexpressed across the dataset (gene modules). These gene modules were identified in an unbiased manner through hierarchical clustering of gene-gene Spearman correlation coefficient ${ }^{18}$.Gene modules of interest were selected based on the presence of well-characterized makers for placodal, neural and neural-crest cells including the new otic or epibranchial genes identified above (Fig.1d). Initial cell clustering defines five major clusters (Extended Data Fig. 2a) to which we assigned identities using known markers (Fig. 1g-h, Extended Data Fig. 2c-f). Clusters C1 and C2 represent $\mathrm{Pax} 2^{+} / \mathrm{Six} 1^{+}$cells expressing high levels of OEP and placodal makers (Fig. 1h, i; Extended Data Fig. 2c). Surprisingly, we also find two clusters with relatively few $\mathrm{Pax}^{+}$cells: one containing neural-like cells $\left(\mathrm{C} 4\right.$; Sox $\left.21^{+}\right)$and another containing neural crest-like cells $\left(\mathrm{C} 5 ; \mathrm{Pax} 7^{+}\right)($Extended Data Fig. 2a, d, e). Since OEPs are mixed with neural and neural crest cells at early stages ${ }^{9}$, this observation 
suggests that while cells in clusters C4/5 initially activate Pax2E1-EGFP they subsequently downregulate enhancer activity and Pax2 expression.

To investigate the transcriptional dynamics accompanying otic and epibranchial fate decisions, we subset the placodal clusters (C1/2 in Fig. 1; Extended Data Fig. 2a). Re-clustering these cells using gene modules containing otic and epibranchial genes we obtained five placodal clusters (PC1-5; Fig. 1m, j, k; Supplementary Table 2). PC1 largely consists of only OEPs (ss8-9 cells), while the other clusters contain cells from both early and late placode stages (Fig.1m, j, k). Indeed, PC1 is characterised by the expression of OEP genes (GM1-3; Fig. 1m), as well as sharing genes with the otic module GM5/6 and the epibranchial module GM7-9. In contrast, PC2/3 and PC4/5 are transcriptionally distinct from each other with profiles akin to otic (GM5/6) and epibranchial (GM7-9) cells, respectively. To explore the relationship between different cell clusters we organised cells along pseudo-time using Monocle $2^{19}$. This analysis predicts that OEPs gradually split into one otic and one epibranchial branch, each composed of early and late placodal cells (Fig.1n, o). Calculating RNAvelocity independently ${ }^{20}$ and embedding the corresponding vector field onto the Monocle2 trajectory validates the directionality of predicted cell state transitions (Fig.1p).

To explore dynamic changes of gene expression accompanying these inferred trajectories, we used Branch-Expression-Analysis-Modelling (BEAM) ${ }^{19}$. This identified groups of transcription factors expressed in OEPs prior to the branching point, which subsequently segregate into either the otic (e.g. Sox8, Lmx1a) or the epibranchial (e.g. Foxi3, Tfap2e) branch (Fig. 2a, Extended Data Fig. 3a). To quantify the changes in co-expression of otic and epibranchial genes, we assessed the proportion of co-expressing cells before and after the branching point. A two-tailed Wilcoxon rank sum test reveals significantly more cells co-expressing otic and epibranchial markers in OEPs than in epibranchial $(W=214, p=0.0013)$ and otic cells $(W=235, p<0.0001)$ after the branching point (Fig. $2 b$, c). Quantification of gene expression in the monocle trajectories and by in situ hybridization chain reaction $(\mathrm{HCR})^{21}$ confirms that otic (Sox8, Lmx1a) and epibranchial (Foxi3, Tfap2e) transcripts overlap at ss8-9, and their expression resolves as both placodes are firmly established (Fig. 2d-i, Extended Data Fig. 3b-f). 
Together, these results identify groups of transcription factors whose expression changes over time as otic and epibranchial precursors segregate. Some of these may play a key role in imparting ear identity.

\section{Epigenomic profiling uncovers new regulatory elements and motifs in ear precursors}

Transcription factors controlling cell fate choice regulate cell-specific downstream targets by interacting with tissue specific cis-regulatory enhancer elements. Active enhancers are regions of open chromatin flanked by nucleosomes enriched for histone 3 lysine-27 acetylation (H3K27ac), while actively transcribed genes are marked by $\mathrm{H} 3 \mathrm{~K} 4 \mathrm{me} 3^{22-25}$. We therefore profiled ss8-9 OEPs by ChIPseq for H3K27ac, H3K4me3 and the repressive mark H3K27me3 and determined chromatin accessibility by ATACseq. Overlapping H3K27ac and ATACseq data identifies 10969 regions with depleted H3K27me3 marks; average profiles show bimodal H3K27ac read distribution surrounding ATACseq peaks (Extended Data Fig. 4a). Of these just over $70 \%$ are intergenic or intronic representing putative enhancers (8316), while the remaining are close to transcription start sites (TSS; Extended Data Fig. 3b). We associated each putative enhancer to the nearest TSS of protein coding genes; GO term analysis of the corresponding genes returns MAP-kinase, Wnt and Notch signalling known to mediate ear induction, development and neurogenesis (Extended Data Fig. 4c) ${ }^{13,15}$. We selected putative enhancers in the vicinity of ear-enriched genes to assess their activity in vivo, generated EGFP reporters and co-electroporated them with ubiquitously expressed mCherry into head-fold-stage chick embryos. RT-PCR-based assays ${ }^{26}$ (not shown) and fluorescence microscopy confirm enhancer activity in ear progenitors and otic placodes (Fig. 3a, b; Extended Data Fig. 5-7). Next, we performed motif enrichment analysis of all ear enhancers to identify upstream regulators that may act as otic determinants. This reveals an over-representation of binding sites for Sox, TEAD and Six family members and for Tfap2a (Fig. 3c; Extended Data Fig. 4d). Of these, Tfap2a and Six proteins have previously been implicated in cranial placode development ${ }^{27-31}$, confirming that this strategy can identify relevant regulatory factors.

We also exploited the idea that cell identity genes may be regulated by super-enhancers 
OEP factors segregating to the ear lineage (Fig. 2a) that are also prominent in the otic $\mathrm{GRN}^{16}$, we find that the Sox8 locus is marked with H3K4me3, while enhancers close to Lmx1a, Zbtb16 and Sox13 show broad H3K27ac marks (Fig. 3a, Extended data 4e, 6a, 7a). Together, this analysis points to Lmx1a, Zbtb16 and members of the Sox transcription factor family as potential key regulators of ear identity.

\section{Sox8, Lmx1a and Pax2 are core components of the ear determination network}

Our transcriptome analysis identifies several Sox genes enriched in otic progenitors. Of these, Sox3 and Sox13 are expressed prior to ear specification, while Sox 9 and -10 are activated later ${ }^{16}$. These genes are therefore unlikely to initiate the otic programme. In contrast, the SoxE group factor Sox8 is highly enriched in OEPs prior to segregating to otic cells (Fig. 3d). In situ hybridisation reveals that Sox8 begins to be expressed at 3ss, followed shortly thereafter by Pax2, Zbtb16, and $L m \times 1 a$, while Foxg1, Soho1 and Sox10 are activated later (Fig. 3d, Extended Data Fig. 5a).

To explore the regulatory interactions between the earliest OEP transcription factors, we systematically knocked down each one in turn and assayed the expression of all others as well as that of Foxg1 and Soho1 as a readout for otic identity using in situ hybridisation and RT-qPCR (Fig. 2e; Extended Data Fig. 5b, c). Control or antisense oligonucleotides targeting Sox8, Pax2, Zbtb16 or Lmx1a were electroporated into future OEPs of head-fold-stage chick embryos and gene expression analysed at OEP stage (ss8-9). We find that Sox8 is necessary for the expression of all assayed ear transcription factors. Pax2 is required for the expression of Zbtb16 and $L m \times 1 a$, which in turn is necessary for Pax2, suggesting that they act in a positive feedback loop. All gene expression changes can be rescued by co-electroporation of the appropriate full-length constructs (Extended Data Fig. 9). These experiments put Sox8 at the top of the ear determination network forming a regulatory circuit with Pax2 and Lmx1a.

\section{Sox8 induces ectopic otic vesicles and vesicle-derived neurons}

If Sox8, Pax2 and Lmx1a indeed form a minimal circuit driving otic specification, they should be able to convert non-ear cells into cells with ear identity. We tested this hypothesis by electroporating a 
combination of Sox8/Pax2/Lmx1a-mCherry-tagged constructs into head-fold-stage ectoderm not destined to contribute to the ear. After $72 \mathrm{hrs}$ we observe many otic vesicles scattered across the head ectoderm. Some of these contain neurofilament-positive neurons, with processes projecting out from the ectopic vesicle (Fig. 4a).

We next asked whether Sox8 alone can initiate the otic programme using the Lmx1aE1-EGFP enhancer and otic markers as a readout. We find that misexpression of Sox8-mCherry not only activates Lmx1aE1-EGFP in ectopic vesicles, but also the expression of Pax2 and $L m \times 1 a$, and the ear marker Soho-1 (Fig. 4b-d; Extended Data Fig. 10a). To assess to what extent Sox8 can confer ear identity, we isolated double-positive Sox8-mCherry/Lmx1a-EGFP cells from ss11-12 by FACS and compared their transcriptome with control ectoderm labelled with constitutive mCherry/EGFP. Differential expression analysis shows upregulation of 399 transcripts in comparison to controls, while 112 genes are downregulated ( $\log 2 \mathrm{FC}>1.5$; adjusted p-value < 0.05) (Fig. 4e, Extended Data Fig.

$1739 \mathrm{~b}-\mathrm{d}$, Supplementary Table 3). Seventeen of the twenty seven upregulated transcription factors are known to be expressed in the otic placode or vesicle, while for the remaining ten no expression data are available (Supplementary Table 3). In contrast, among the downregulated genes are typical neural crest and forebrain transcription factors. The first cell types specified during ear formation are neurons of the cochlear-vestibular ganglion, we therefore asked whether Sox8 can also trigger neurogenesis. We find that Sox8-induced ectopic vesicles are associated with neurofilament-positive neurites generated from Sox8 expressing cells themselves (Fig. 4f, g). Together, these results show that Sox8 can activate the transcriptional program for ear identity in non-ear cells by direct lineage reprograming in vivo.

\section{Conclusion}

We have established an integrated workflow for multi-omics data analysis to ensure accessibility and reproducibility. Using transcriptional profiling at single cell level, enhancer discovery and analysis, together with functional experiments, we have identified the critical components of the ear determination network: Sox8, Pax2 and Lmx1a (Fig. 4h). Sox8 lies at the top of the transcriptional hierarchy, and alone is sufficient to impart ear identity to cells otherwise destined to form head 
epidermis, other sense organs or cranial ganglia. Previous findings have implicated Pax2 in otic placode development and patterning ${ }^{35-38}$ and Sox8 in regulating ear-specific enhancers ${ }^{39-41}$. Sox proteins cooperate with a variety of transcription factors to exert their cell type specific function ${ }^{42}$. It is therefore tempting to speculate that, in analogy to the eye, where Sox2 cooperates with Pax6 to regulate lens-specific transcription ${ }^{43}$, in the ear Sox8 might partner with Pax2.

Our results also show that OEPs initially express competing transcriptional programmes that resolve over time as otic and epibranchial cell states are established. We capture previously unknown gene modules that accompany this process as well as regulatory regions associated with oticepibranchial specification. In turn, this information is critical to unravel the underlying gene regulatory networks and identify the transcription factor codes that determine cell identity in the cranial sensory nervous system. In the long term, this will enhance our ability to engineer specific sensory cell types for basic research and regenerative purposes.

\section{References}

2031 Jacobson, A. G. The determination and positioning of the nose, lens, and ear. III. Effects of reversing the antero-posterior axis of epidermis, neural plate and neural fold. $J$ Exp Zool 154, 293-303 (1963).

Groves, A. K. \& Bronner-Fraser, M. Competence, specification and commitment in otic placode induction. Development 127, 3489-3499 (2000). olfactory placode fate. Development 135, 4165-4177 (2008). Rev Dev Biol 3, 349-363, doi:10.1002/wdev.142 (2014).

2146 Thiery, A., Buzzi, A. L. \& Streit, A. Cell fate decisions during the development of the peripheral nervous system in the vertebrate head. Curr Top Dev Biol 139, 127-167, doi:10.1016/bs.ctdb.2020.04.002 (2020). 
2177 Davis, R. L., Weintraub, H. \& Lassar, A. B. Expression of a single transfected cDNA converts 218 fibroblasts to myoblasts. Cell 51, 987-1000, doi:10.1016/0092-8674(87)90585-x (1987).

2198 Gehring, W. J. The master control gene for morphogenesis and evolution of the eye. Genes Cells 1, 11-15. (1996).

Streit, A. Extensive cell movements accompany formation of the otic placode. Dev Bio/ 249, 237-254 (2002).

Pieper, M., Eagleson, G. W., Wosniok, W. \& Schlosser, G. Origin and segregation of cranial placodes in Xenopus laevis. Dev Biol 360, 257-275, doi:S0012-1606(11)01268-1 [pii] 10.1016/j.ydbio.2011.09.024 (2011).

Ladher, R. K., Anakwe, K. U., Gurney, A. L., Schoenwolf, G. C. \& Francis-West, P. H.

Identification of synergistic signals initiating inner ear development. Science 290, 1965-1968. (2000).

Wright, T. J. \& Mansour, S. L. Fgf3 and Fgf10 are required for mouse otic placode induction. Development 130, 3379-3390 (2003).

Ohyama, T., Groves, A. K. \& Martin, K. The first steps towards hearing: mechanisms of otic placode induction. Int J Dev Biol 51, 463-472 (2007).

Ladher, R. K., O'Neill, P. \& Begbie, J. From shared lineage to distinct functions: the development of the inner ear and epibranchial placodes. Development 137, 1777-1785, doi:137/11/1777 [pii] 10.1242/dev.040055 (2010).

Chen, J. \& Streit, A. Induction of the inner ear: stepwise specification of otic fate from multipotent progenitors. Hear Res 297, 3-12, doi:10.1016/j.heares.2012.11.018 (2013).

16 Chen, J. et al. A systems-level approach reveals new gene regulatory modules in the developing ear. Development 144, 1531-1543, doi:10.1242/dev.148494 (2017).

7 Nishimura, N. et al. A Systematic Survey and Characterization of Enhancers that Regulate Sox3 in Neuro-Sensory Development in Comparison with Sox2 Enhancers. Biology (Basel) 1, 714-735, doi:10.3390/biology1030714 (2012). 
Delile, J. et al. Single cell transcriptomics reveals spatial and temporal dynamics of gene expression in the developing mouse spinal cord. Development 146, doi:10.1242/dev.173807 (2019).

19 Trapnell, C. et al. The dynamics and regulators of cell fate decisions are revealed by pseudotemporal ordering of single cells. Nat Biotechnol 32, 381-386, doi:10.1038/nbt.2859 (2014).

20 La Manno, G. et al. RNA velocity of single cells. Nature 560, 494-498, doi:10.1038/s41586018-0414-6 (2018).

21 Choi, H. M. T. et al. Third-generation in situ hybridization chain reaction: multiplexed, quantitative, sensitive, versatile, robust. Development 145, doi:10.1242/dev.165753 (2018).

Creyghton, M. P. et al. Histone H3K27ac separates active from poised enhancers and predicts developmental state. Proc Natl Acad Sci U S A 107, 21931-21936, doi:10.1073/pnas.1016071107 (2010).

23 Kharchenko, P. V. et al. Comprehensive analysis of the chromatin landscape in Drosophila melanogaster. Nature 471, 480-485, doi:10.1038/nature09725 (2011).

Rada-Iglesias, A. et al. A unique chromatin signature uncovers early developmental enhancers in humans. Nature 470, 279-283, doi:nature09692 [pii] 10.1038/nature09692 (2011).

Zentner, G. E., Tesar, P. J. \& Scacheri, P. C. Epigenetic signatures distinguish multiple classes of enhancers with distinct cellular functions. Genome Res 21, 1273-1283, doi:10.1101/gr.122382.111 (2011).

26 Chen, J. \& Streit, A. A medium-scale assay for enhancer validation in amniotes. Dev Dyn 244, 1291-1299, doi:10.1002/dvdy.24306 (2015).

Brugmann, S. A., Pandur, P. D., Kenyon, K. L., Pignoni, F. \& Moody, S. A. Six1 promotes a placodal fate within the lateral neurogenic ectoderm by functioning as both a transcriptional activator and repressor. Development 131, 5871-5881 (2004). 
26928 Ozaki, H. et al. Six1 controls patterning of the mouse otic vesicle. Development 131, 551$270 \quad 562(2004)$

Zheng, W. et al. The role of Six1 in mammalian auditory system development. Development 130, 3989-4000 (2003).

Maharana, S. K. \& Schlosser, G. A gene regulatory network underlying the formation of preplacodal ectoderm in Xenopus laevis. BMC Biol 16, 79, doi:10.1186/s12915-018-0540-5 (2018).

Kwon, H. J., Bhat, N., Sweet, E. M., Cornell, R. A. \& Riley, B. B. Identification of early requirements for preplacodal ectoderm and sensory organ development. PLoS Genet 6 , doi:10.1371/journal.pgen.1001133 (2010).

Benayoun, B. A. et al. H3K4me3 breadth is linked to cell identity and transcriptional consistency. Cell 158, 673-688, doi:10.1016/j.cell.2014.06.027 (2014).

Hnisz, D. et al. Super-enhancers in the control of cell identity and disease. Cell 155, 934947, doi:10.1016/j.cell.2013.09.053 (2013).

Whyte, W. A. et al. Master transcription factors and mediator establish super-enhancers at key cell identity genes. Cell 153, 307-319, doi:10.1016/j.cell.2013.03.035 (2013).

Torres, M., Gomez-Pardo, E. \& Gruss, P. Pax2 contributes to inner ear patterning and optic nerve trajectory. Development 122, 3381-3391 (1996).

Burton, Q., Cole, L. K., Mulheisen, M., Chang, W. \& Wu, D. K. The role of Pax2 in mouse inner ear development. Dev Biol 272, 161-175 (2004).

37 Christophorou, N. A., Mende, M., Lleras-Forero, L., Grocott, T. \& Streit, A. Pax2 coordinates epithelial morphogenesis and cell fate in the inner ear. Dev Biol 345, 180-190, doi:S00121606(10)00911-5 [pii] 10.1016/j.ydbio.2010.07.007 (2010).

Freter, S. et al. Pax2 modulates proliferation during specification of the otic and epibranchial placodes. Dev Dyn 241, 1716-1728, doi:10.1002/dvdy.23856 (2012). 
29439 Betancur, P., Sauka-Spengler, T. \& Bronner, M. A Sox10 enhancer element common to the 295 otic placode and neural crest is activated by tissue-specific paralogs. Development 138, 3689-3698, doi:dev.057836 [pii] 10.1242/dev.057836 (2011).

40 Murko, C. \& Bronner, M. E. Tissue specific regulation of the chick Sox10E1 enhancer by different Sox family members. Dev Biol 422, 47-57, doi:10.1016/j.ydbio.2016.12.004 (2017).

Okamoto, Y. et al. Cooperation of Sall4 and Sox8 transcription factors in the regulation of the chicken Sox3 gene during otic placode development. Dev Growth Differ 60, 133-145, doi:10.1111/dgd.12427 (2018).

Kondoh, H. \& Kamachi, Y. SOX-partner code for cell specification: Regulatory target selection and underlying molecular mechanisms. The International Journal of Biochemistry \& Cell Biology 42, 391-399 (2010).

Kamachi, Y., Uchikawa, M., Tanouchi, A., Sekido, R. \& Kondoh, H. Pax6 and SOX2 form a co-DNA-binding partner complex that regulates initiation of lens development. Genes Dev $15,1272-1286$ (2001). 
a
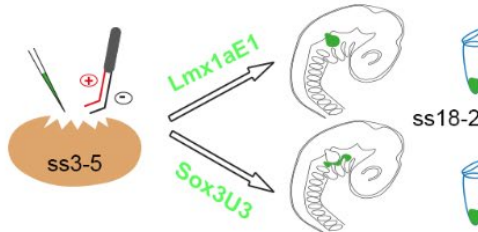

e

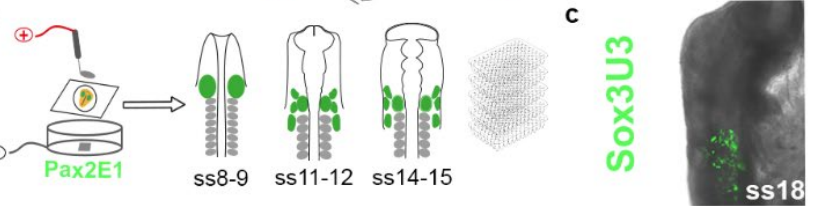

$\mathbf{f}$

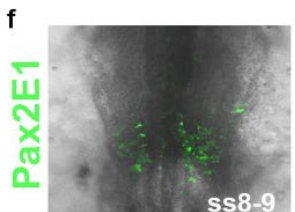

b
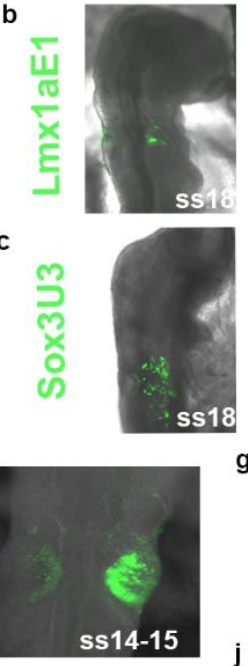

m

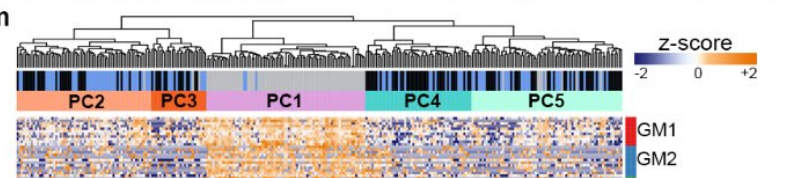

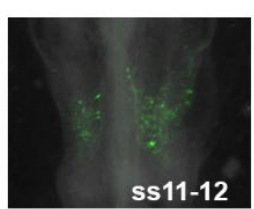

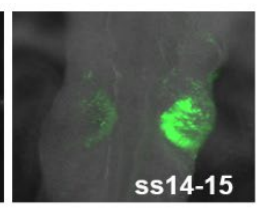

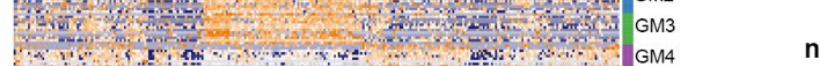

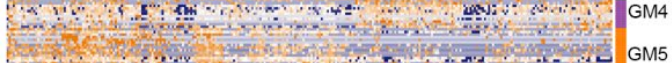

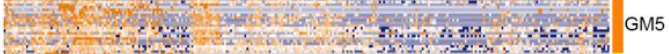

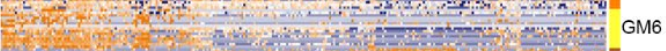

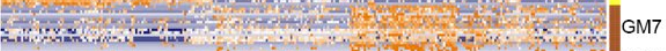
(2) Atrits

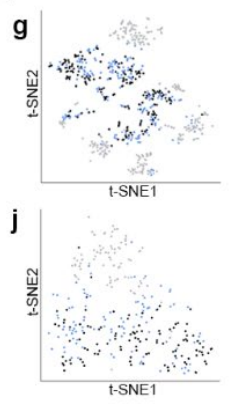

n

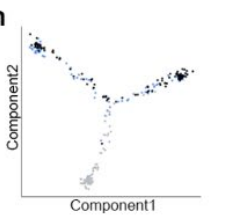

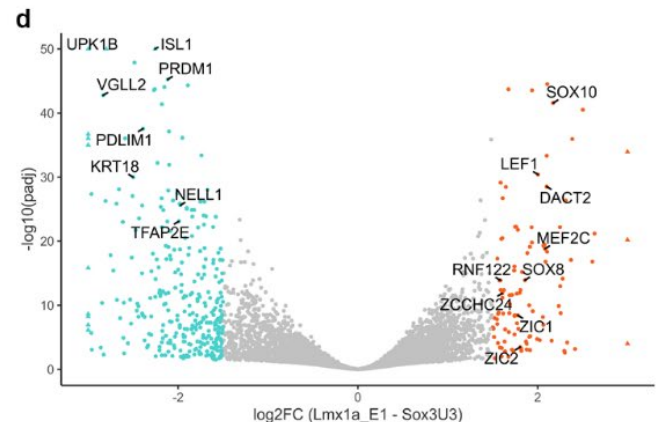
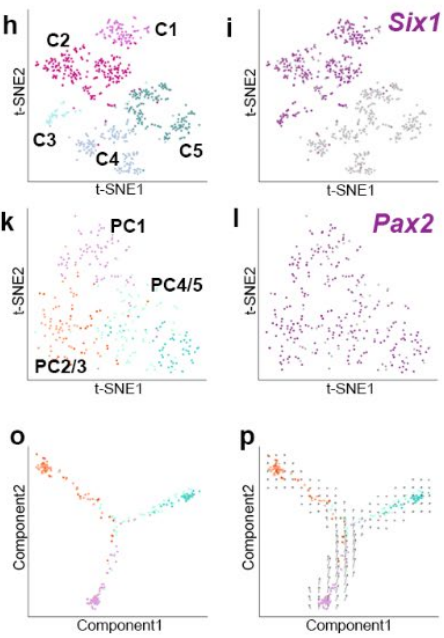

322 Figure 1. Transcriptomic characterisation of ear development. a-c, In ovo electroporation (a) was used to label and collect otic (b; Lmx1aE1-EGFP+) and epibranchial (c; Sox3U3-EGFP+) cells for bulk RNAseq. d, Volcano plot showing genes differentially expressed (absolute log2 fold-change $>1.5$ and adjusted $p$-value $<0.05$ ) in otic (orange) and epibranchial cells (green). e, f, Cells were collected for scRNAseq after ex ovo electroporation of the Pax2E1-EGFP enhancer, which is active in OEPs, otic and epibranchial placodes (f); cells were collected at the stages indicated. g-I, tSNE representation of unsupervised hierarchical clustering of all cells $(\mathbf{g}-\mathbf{i})$ and of the placodal cell subset (j-I); cells were colour-coded according to stage collected (g, j; OEP grey, ss11-12 blue, ss14-15 black), clusters (h, k) and placodal markers Six1 (i) and Pax2 (I) expression. m, Heatmap showing partitioning of the placodal subset $(\mathrm{C} 1, \mathrm{C} 2$ in $\mathrm{h})$ into five clusters (PC1-5) based on gene modules (GM). Expression profiles reveal that PC1 largely contains OEP-like cells, while PC2/ and PC4/5 are composed of otic-

333 like and epibranchial-like cells. n-o, Pseudo-time ordering using Monocle2 shows trajectories 334 between stages $(\mathbf{n})$ and clusters (o). Note: otic- and epibranchial-like cells segregate into two branches. p, RNAvelocity vector field verifies the directional trajectories predicted by Monocle2. 

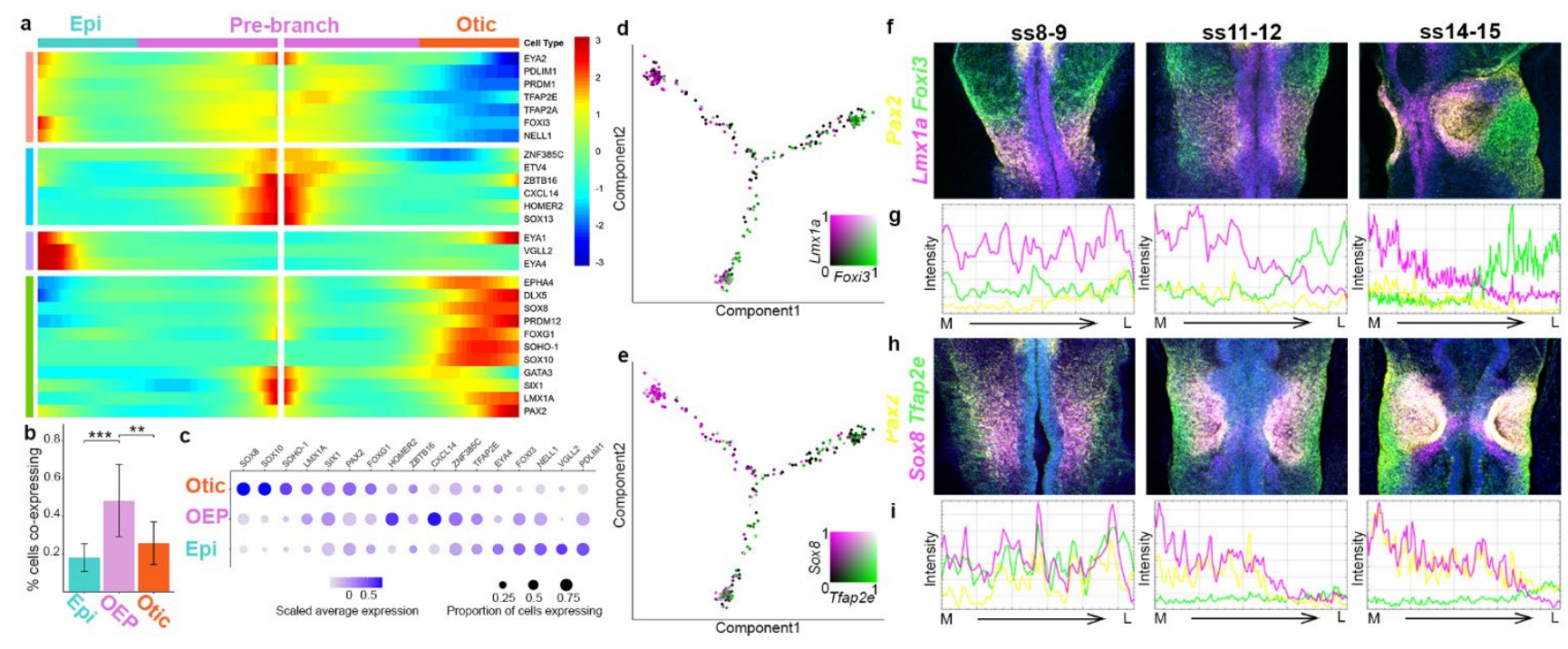

Figure 2. Dynamic gene expression during OEP segregation into otic and epibranchial

lineages. a, BEAM identifies genes regulated in a branch-dependant manner. b, Histogram showing the proportion of cells co-expressing genes that are expressed before the branching point, but segregate to the otic or epibranchial branch. Significantly more cells co-express such genes before the branching point than thereafter. Error bars indicate $+/-1$ standard deviation. ${ }^{* *} p$-value $<0.01 ;{ }^{* * *}$ p-value $<0.001$. c, Dot plot for OEP, otic and epibranchial markers based on scRNAseq data. Expression level is indicated by colour intensity and gene expression frequency by dot size. d, e, A proportion of cells co-expresses otic (Sox8, Lmx1a) and epibranchial (Foxi3, Ap2e) markers prior to the branching point. $\mathbf{f - i}$, In situ $\mathrm{HCR}(\mathbf{f}, \mathbf{h})$ and intensity measurements $(\mathbf{g}, \mathbf{i})$ along the medial $(\mathrm{M})$ to lateral $(\mathrm{L})$ axis of the OEP-domain confirms gradual segregation of OEP gene expression as distinct otic and epibranchial cell populations emerge. 


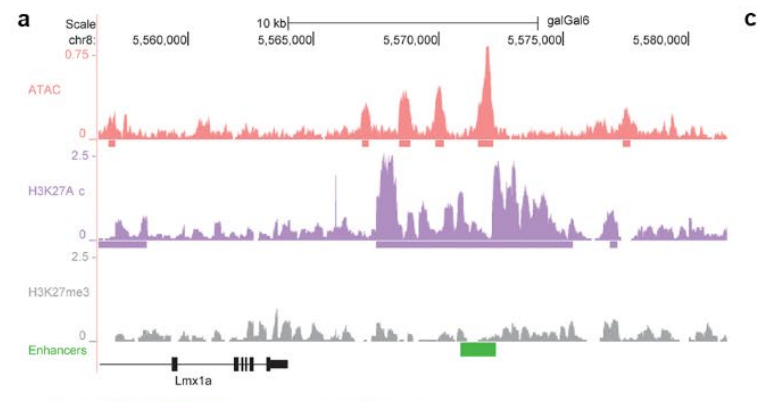

b Lmx1aE1-EGFP

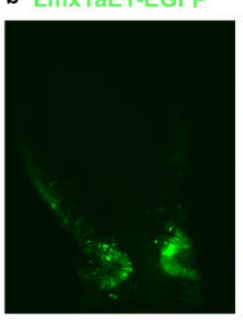

mCherry

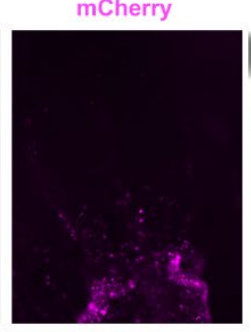

Merge

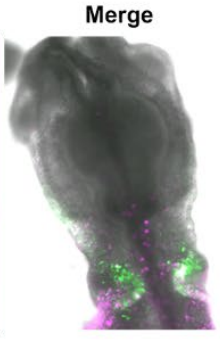

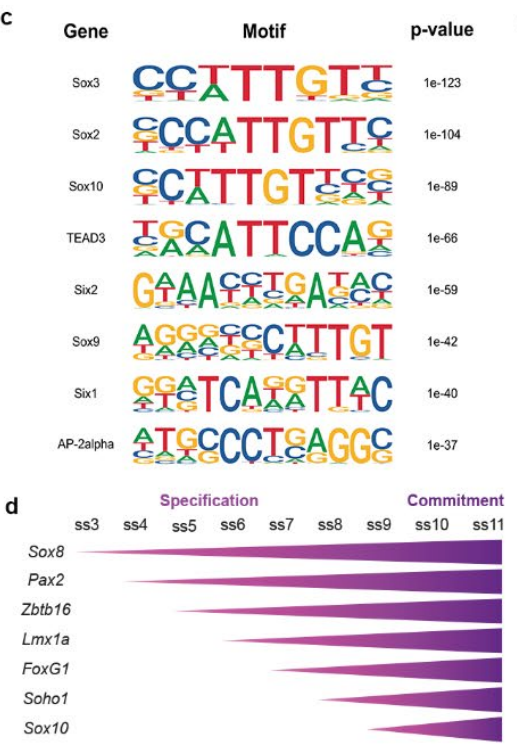

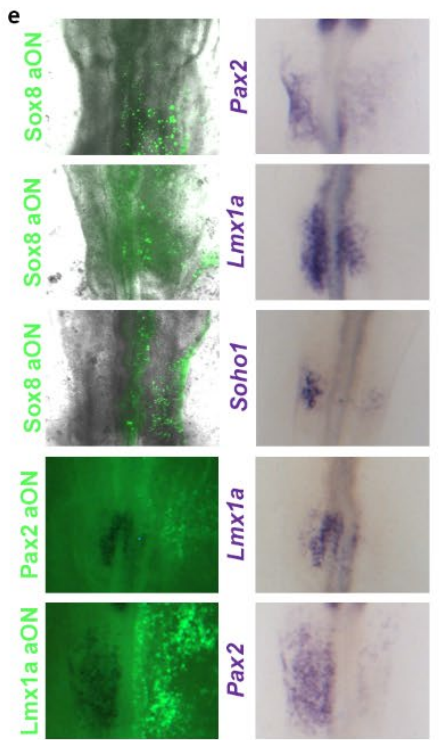

Figure 3. Chromatin accessibility profile of OEPs identifies putative regulatory regions. a,

Genome browser view of ATAC, H3K27ac and H3K27me3 profiles in OEPs at the Lmx1a locus.

Cloned putative enhancer is shown in green. $\mathbf{b}$, Coelectroporation of Lmx1aE1-EGFP reporter (green)

and constitutive mCherry (magenta) reveals in vivo enhancer activity in the otic placode. c, Motif

enrichment analysis of all identified enhancers. d, Diagram showing the activation of expression of

potential regulators of otic identity during specification and commitment stages. e, Knock-down of

selected transcription factors using fluorescein labelled antisense oligonucleotides (aON; green) lead

to downregulation of otic markers as identified by in situ hybridisation (blue). 

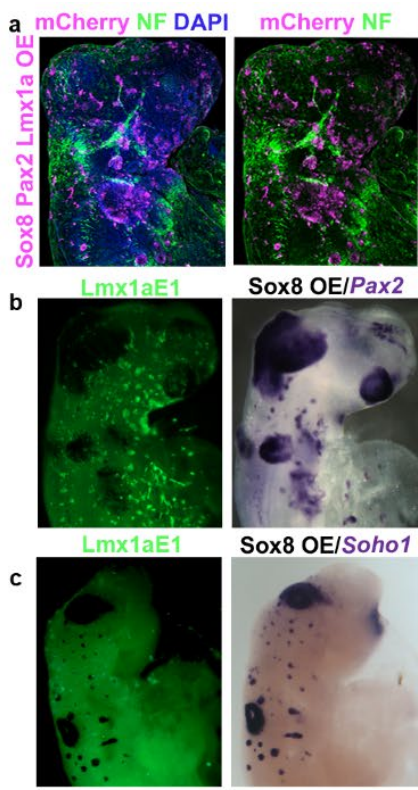

Sox8 OEIPax2

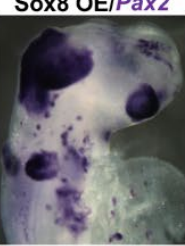

Sox8 OE/Soho1
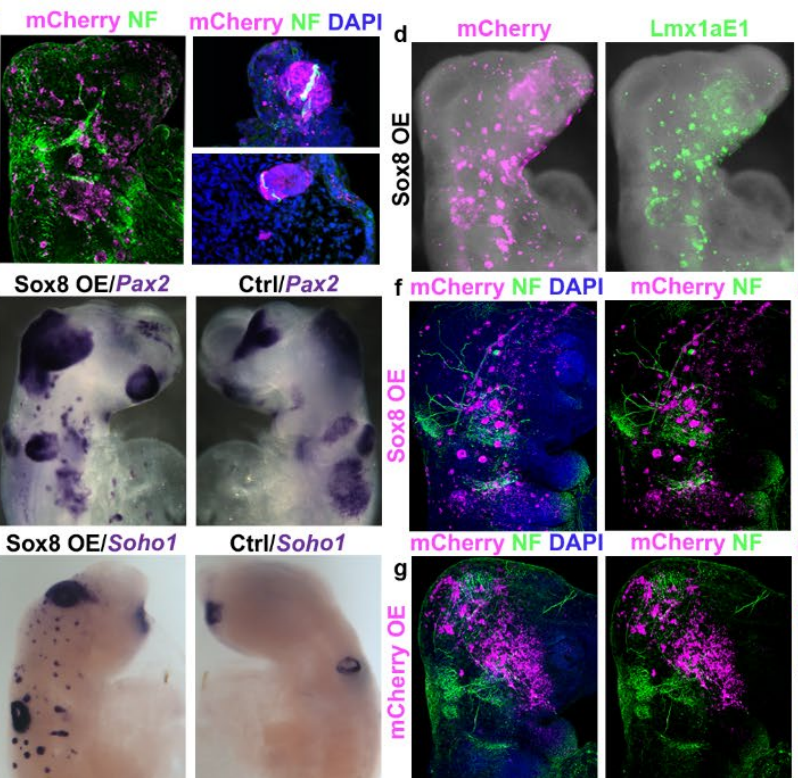

$\mathrm{Ctrl} / \mathrm{Pax} 2$

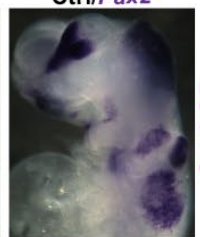

Ctrl/Soho1
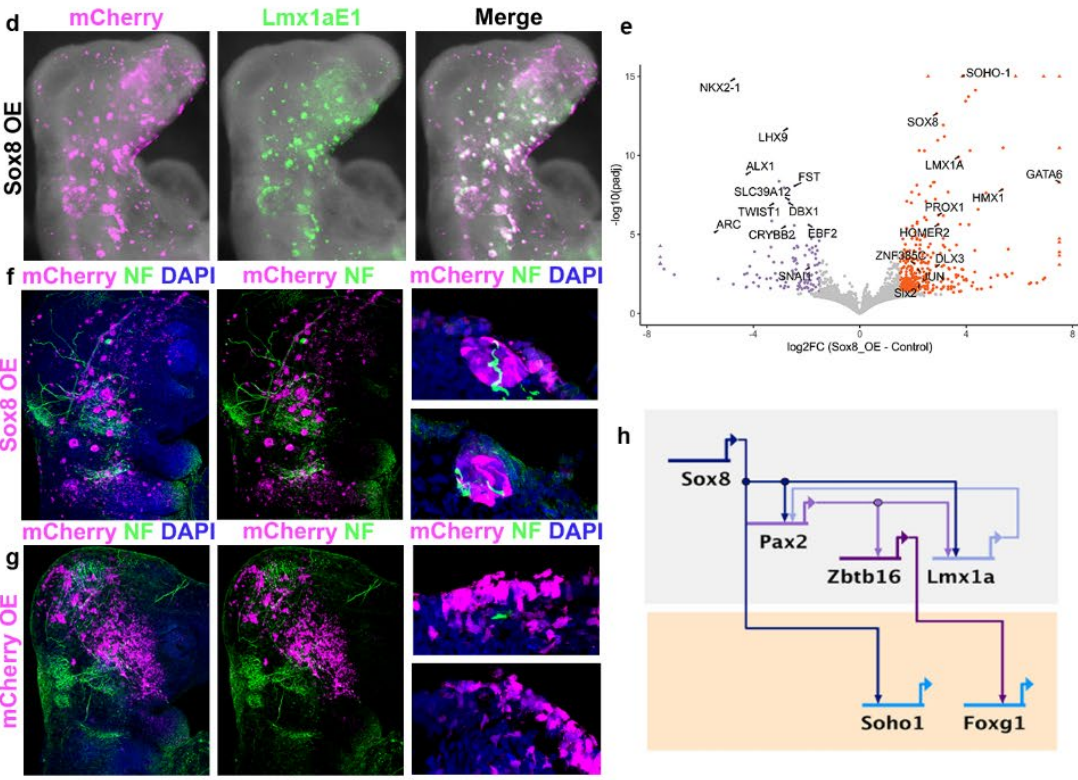

Figure 4. Direct reprogramming of ectoderm by Sox8. a, Co-electroporation of mCherry-tagged

vectors containing the full-length sequence of Sox8/Pax2/Lmx1a (magenta) generate ectopic

vesicles across the cranial ectoderm after $72 \mathrm{hrs}$ culture. Neurofilament antibody staining (NF; green) shows axonal projections from the vesicle. b-d, Overexpression of Sox8 (Sox8 OE) alone activates Lmx1aE1-EGFP (green) and otic marker expression on the electroporated, but not on the control side (Ctrl). e, RNAseq of Sox8 and control electroporated cranial ectoderm; volcano plot shows enrichment of otic genes in the Sox 8 OE (absolute log2 fold-change $>1.5$ and adjusted $p$ value $<0.05)$. f, Sox8-mCherry overexpression (magenta) alone generates ectopic otic vesicles with neuronal projections (green), while controls do not $(\mathbf{g}) \cdot \mathbf{h}$, BioTapestry model showing the minimal 


\section{Methods}

\section{Expression and enhancer constructs}

374 Putative enhancers were amplified from chick genomic DNA and cloned into pTK-EGFP reporter 375 vectors after digestion with $\mathrm{Xcml}^{26}$. To generate expression constructs total RNA was isolated from 376 HH8-12 chick embryos with RNAqueous-Micro Total RNA Isolation Kit (Thermo Fisher Scientific), 377 reverse transcribed using Superscript II reverse transcriptase (Thermo Fisher Scientific, 180644-014) and oligo-dT primer. Specific primers were used to amplify the full-length coding sequence of Sox8, Pax2, Lmx1a and Zbtb16 and PCR products were cloned into pCAB-IRES-mCherry. All sequences were verified by Sanger sequencing.

\section{Chick embryos, electroporation and culture}

Fertilised hens' eggs (Stewart, Norfolk UK) were incubated at $38^{\circ} \mathrm{C}$ and staged according to Hamburger and Hamilton $(\mathrm{HH})^{44}$. All experiments were performed on embryos younger than 12 days, thus were not regulated by the Animals Scientific Procedures Act 1986.

OEPs for bulk RNAseq were labelled using in ovo electroporation ${ }^{45}$; eggs were incubated until

the 3-6 somite stage (ss), pTK-Lmx1aE1-EGFP or pTK-Sox3U3-EGFP plasmids $(1 \mu \mathrm{g} / \mu \mathrm{l})$ were injected targeting the OEP territory. Electroporation using Ovodyne electroporator (TSS20, Intracel) was performed with five $50 \mathrm{~ms}$ pulses of $8 \mathrm{~V}$ at $100 \mathrm{~ms}$ intervals. After incubation until ss $18-21$, embryos were collected in PBS and processed for bulk RNAseq.

For ex ovo culture, embryos were harvested using filter paper rings ${ }^{46}$ and cultured on egg albumen; for long-term culture (72hrs) the 'modified Cornish pasty' method ${ }^{47}$ was used. Ex ovo electroporation was performed to collect cells for scRNAseq, for knock-down, rescue and overexpression experiments. The posterior placodal region or the cranial surface ectoderm of $\mathrm{HH} 6$ embryos was

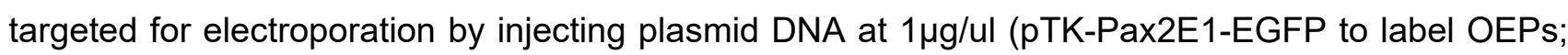
pCES-Sox8-mCherry + pTK-Lmx1aE1-EGFP, pCES-Sox8-mCherry + pCES-Lmx1a-mCherry + pCES-Pax2-mCherry; pCES-mCherry + pCES-EGFP for misexpression), control or antisenseoligonucleotides (aON; GeneTools; $0.75 \mathrm{mM}$ with $0.3 \mu \mathrm{g} / \mu \mathrm{l}$ carrier DNA), or a combination of aON and 
401 Lmx1a:

5'-CCTCCATCTTCAAGCCGTCCAGCAT-3'16,

Zbtb16:

5 GTCAAATCCATAGCACTCCCGAGGT-3', Sox13: 5'-CTCCTCATGGACATCCATTTCATTC-3' and control 5'-ATGGCCTCGGAGCTGGAGAGCCTCA-3'. Electroporation was performed in a chamber using five $5 \mathrm{~V}$ pulses of $50 \mathrm{~ms}$ in $100 \mathrm{~ms}$ intervals ${ }^{45}$.

405

\section{Whole mount and hybridization chain reaction fluorescent in situ hybridisation}

In situ hybridization was carried out following previously described protocols ${ }^{48}$. Whole-mount pictures were taken using an Olympus SZX12 with a Retiga2000R camera and Q-Capture Pro7 software.

HCR v3 was performed using the Molecular Technologies protocol ${ }^{21}$. Briefly, embryos were fixed in 4\% PFA for one hour at room temperature, dehydrated in a series of methanol in PBT and stored overnight at $-20^{\circ} \mathrm{C}$. After rehydration and proteinase- $\mathrm{K}$ treatment $(20 \mathrm{mg} / \mathrm{ml} ; 3 \mathrm{~min})$ embryos were post-fixed in 4\% PFA for 20 min. Embryos were then washed on ice in PBS, 1:1 PBT/5X SSC (5X sodium chloride sodium citrate, $0.1 \%$ Tween-20) and 5X SSC for 5 min each. Pre-hybridization in hybridisation buffer was performed for 5 min on ice, followed by 30 minutes at $37^{\circ} \mathrm{C}$. Embryos were hybridised overnight at $37^{\circ} \mathrm{C}$ with probes at $4 \mathrm{pmol} / \mathrm{ml}$ in hybridization buffer. After four 15 min washes with probe wash buffer at $37^{\circ} \mathrm{C}$, preamplification was carried out in amplification buffer for 5 min at room temperature. Hairpins were prepared individually at $30 \mathrm{pmol}$ final concentration; they were incubated at $95^{\circ} \mathrm{C}$ for 90 seconds followed by cooling to room temperature for $30 \mathrm{~min}$, protected from light. Cooled hairpins were added to $500 \mu$ amplification buffer, embryos were incubated in hairpins overnight at room temperature followed by two $5 \mathrm{~min}$ and two $30 \mathrm{~min}$ washes in $5 \mathrm{X}$ SSC. After a 5 min incubation in DAPI $(10 \mathrm{mg} / \mathrm{ml})$, they were washed three times for 10 min with $5 X \mathrm{SSC}$, before being imaged using Leica SP5 laser scanning confocal inverted microscope using the LAS AF software.

For HCR image analysis, Z-stacks were collected for $50-70 \mu \mathrm{m}$; figures show projections of all stacks. Images were processed using ImageJ and the ImageJ Plot Profile tool was used to calculate intensity plots. In brief, an optical section in the centre of the placode territory was selected using the 
427 Pax2 channel as a reference. The Pax2 channel was added to the Sox8, Lmx1a, Foxi3 or Tfap2e 428 channels, respectively. Intensity values were then calculated across the area of interest and plotted.

\section{Whole mount immunostaining}

431 Embryos were collected in PBS, fixed for 25 minutes at room temperature in 4\% PFA, washed in 432 PBS-Tx (PBS $+0.2 \%$ Triton $\mathrm{X}-100)$ and blocked in $5 \%$ goat serum in PBS-Tx for $3-5 \mathrm{~h}$ at room 433 temperature. Embryos were then incubated in primary antibody diluted in blocking buffer for 24-72 434 hours at $4^{\circ} \mathrm{C}$. Primary antibodies were rabbit anti-mCherry (1:200; Abcam ab167453), mouse anti435 NF (1:100; Thermo Fisher Scientific 13-0700), rabbit anti-GFP (1:500; Abcam a11122) or mouse anti 436 GFP (1:1000; Molecular Probes A11120). After five 60 min washes and one overnight wash in PBS$437 \mathrm{Tx}$, embryos were incubated in secondary antibodies $(1: 800)$ at $4^{\circ} \mathrm{C}$ overnight. Secondary antibodies 438 used were goat anti-rabbit IgG Alexa Fluor 488 (Thermo Fisher Scientific, A11036), donkey anti439 rabbit Alexa Fluor 568 (Thermo Fisher Scientific, A11001), goat anti mouse IgG Alexa Fluor 488 440 (Molecular Probes A11001) and goat anti mouse IgG Alexa Fluor 568 (Molecular Probes A11004). 441 Embryos were then were briefly incubated in PBS containing $10 \mathrm{mg} / \mathrm{ml} \mathrm{DAPI}$ and washed at least five 442 times in PBS-Tx before being mounted on slides and imaged using Leica SP5 laser scanning confocal 443 inverted microscope using a 10x objective or an Olympus SZX12 with a Retiga2000R camera and Q444 Capture Pro7 software. Confocal whole mount images in the manuscript are maximum intensity projections of embryo z-stacks. Sections were imaged using a $63 x$ oil immersion objective and maximum intensity projections are shown.

\section{Cryosectioning}

449 Embryos were embedded in gelatine as previously described ${ }^{49}$ and cryo-sectioned at $15-20 \mu m$ using 450 a Bright OTF5000 cryostat. Sections were mounted using Mowiol 4-88 (Sigma Aldrich, 81381) and 451 imaged using Leica SP5 laser scanning confocal inverted microscope (LAS AF software) or a Zeiss 452 Axiovert 200M microscope with a Hamamatsu C4742-95 camera and using OCULAR software. 
455 RNA from dissected otic tissue was isolated using the RNAqueous-Micro Kit (Ambion, AM1931) and 456 reverse transcribed. Primers for target genes were designed with PrimerQuest (IDT). qPCR was 457 performed using Aria Mx Real-Time System (Agilent Technologies) with SYBR green master mix 458 (Roche, 64913850001). RT-qPCR for antisense oligonucleotide experiments was carried out with a 459 minimum of three biological replicates; two sample Welch's t-tests with Benjamini-Hochberg multiple test correction were used to determine statistical significance between control and antisense oligonucleotide $\Delta \mathrm{Ct}$ values. Relative expression $\left(2^{-\Delta \Delta C t}\right)^{50}$ was calculated using the NormqPCR package in R. The geometric mean of Gapdh and Rplp1 expression, or Rplp1 expression alone, was used to normalise gene expression.

\section{Bulk RNA sequencing}

To label otic and epibranchial cells embryos were electroporated with Lmx1aE1-EGFP and Sox3U3EGFP plasmids, respectively, and whole heads were used for cell collection. For overexpression experiments embryos were electroporated with Sox8-mCherry+Lmx1aE1-EGFP or with pCABmCherry+pCAB-EGFP, the endogenous otic placode and the trunk were removed before cell dissociation. Cells were dissociated in FACSmax cell dissociation solution (Ambion, T200100) containing papain (30U/mg, Sigma-Aldrich, 10108014001) for $20 \mathrm{~min}$ at $37^{\circ} \mathrm{C}$ before being transferred 473 containing $5 \%$ heat-inactivated foetal-bovine-serum (FBS), rock inhibitor (10 $\mu \mathrm{M}$, Stemcell 474 Technologies, Y-27632) and non-essential amino acids (Thermo Fisher Scientific, 11140035). Cells 475 were disaggregated by pipetting, sequentially filtered through $0.35 \mu \mathrm{m}$ and $0.20 \mu \mathrm{m}$ filters (Miltenyi 476 Biotech, 130-101-812). Pelleted cells were resuspended in 500 $\mu$ HBSS and isolated by fluorescent activated cell sorting (FACS) using a BD FACS-Aria Diva. 2000 cells per biological replicate were collected, centrifuged at $200 x g$ for 5 mins at $4^{\circ} \mathrm{C}$, washed with PBS, and resuspended in lysis buffer. RNA was extracted using Ambion RNAqueous Micro Total RNA isolation kit (AM1931, ThermoFisher Scientific). RNA integrity was checked using Bioanalyser with Agilent RNA 6000 pico kit (Agilent 481 Technologies, 5067-1513); samples with RIN >7 were processed for library preparation. Sequencing libraries were prepared using Nextera Sample low input kit (Illumina, 15028212) and sequenced using 
75bp paired end reads on the Illumina Hiseq4000 platform. A minimum of three biological replicates were used for analysis.

\section{Single-cell RNA extraction, library preparation and sequencing}

HH6-7 embryos were electroporated with Pax2E1-EGFP to label OEPs; cells were dissociated as described above and 288 EGFP $^{+}$cells from each ss8-9, ss11-12 and ss14-15 were collected by FACS in 96 -well plates. Sequencing libraries were prepared following the SmartSeq2 protocol ${ }^{51}$. Libraries were sequenced on the Illumina NextSeq500 platform using single end 75bp sequencing (ss8-9) or on the HiSeq4000 platform using paired end 75bp sequencing (ss11-12, ss14-15).

\section{Nuclei isolation, ATAC library preparation and sequencing}

The ATACseq library was prepared following published protocols ${ }^{52}$. Approximately 30 pieces of the OEP territory were dissected from ss8-9 embryos, dissociated with Dounce homogenizer (tight pestle) in lysis buffer (10mM Tris- $\mathrm{HCl}, \mathrm{pH} 7.4,10 \mathrm{mM} \mathrm{NaCl}, 3 \mathrm{mM} \mathrm{MgCl}_{2}, 0.1 \%$ IGEPAL CA-630). Nuclei were pelleted at $4000 \mathrm{rpm}$ at $4^{\circ} \mathrm{C}$ for $10 \mathrm{~min}$. After removing the lysis buffer, $1.25 \mu \mathrm{l} \mathrm{Tn} 5$ transposase (Illumina, FC-131-1024) were added to $25 \mu \mathrm{l}$ reaction volume and incubated at $37^{\circ} \mathrm{C}$ for 10 mins. Tagmented DNA was then purified with Mini Elute PCR purification kit (Qiagen, 28004) followed by 9 cycles of PCR enrichment using NEB High fidelity PCR kit (NEB, M0541S). The quality of ATAC libraries was assessed with Agilent Bioanalyzer with DNA High Sensitivity kit (Agilent Technologies, 5067-4627) and quantified with Kapa NGS library quantification kit (Kapa Biosystems, KK4824). The libraries were sequenced with Illumina HiSeq 2000/2500 in 2x100 cycles.

\section{H3K27ac, H3K27me3 and H3K4me4 ChIP library preparation and sequencing}

Approximately 200 pieces of OEP ectoderm were dissected from ss8-9 embryos. The tissue was dissociated in nuclei expulsion buffer (0.5\% NP-40, 0.25\% Triton X-100, $10 \mathrm{mM}$ Tris- $\mathrm{HCl}, \mathrm{pH} 7.5$, $3 \mathrm{mM} \mathrm{CaCl}_{2}, 0.25 \mathrm{M}$ sucrose, $1 \mathrm{x}$ protease inhibitor (Roche), $1 \mathrm{mM}$ DTT, and $0.2 \mathrm{mM}$ PMSF) using a Dounce homogenizer (loose pestle); crosslinking was performed in 1\% formaldehyde for 9 minutes, followed by quenching with $1 \mathrm{M}$ glycine for 5 minutes. Cells were then pelleted and washed 3 times 
511 with PBS containing protease inhibitor (Roche, 11873580001). The pellets were snap-frozen and

512 stored at $-80^{\circ} \mathrm{C}$ for later use. Crosslinked chromatin was fragmented by sonication in an ice bath

513 (Misonix Q700 at 7 Amplitude, $5 \times 40$ seconds, 30 seconds on, 60 seconds off) and immunoprecipitated

514 following the Nano-ChIP protocol ${ }^{53}$. For chromatin immunoprecipitation (ChIP), the following 515 antibodies were used anti-H3K27Ac (Abcam, ab4729), anti-H3K4me3 (Diagenode, A5051-001P) and 516 anti-H3K27me3 (Millipore, 07449). Adaptors and primers from NEBNext library preparation kit 517 (Illumina, E6040S) were used to prepare the library following NanoChip protocol ${ }^{53}$. The library was 518 enriched using 14 PCR cycles and quantified with Kapa NGS library quantification kit (Kapa 519 Biosystems, KK4824) before pooling at a concentration of 20nM. Library quality was assessed with 520 Agilent Bioanalyzer with DNA High Sensitivity kit (Agilent Technologies, 5067-4627) and quantified 521 with Kapa NGS library quantification kit (Kapa Biosystems, KK4824). The libraries were sequenced 522 with Illumina HiSeq $2000 / 2500$ in $2 \times 100$ cycles.

\section{High-Throughput-Sequencing-Data (HTSD) Analysis}

525 All data alignment and downstream analysis was carried out using NF-core and custom Nextflow 526 pipelines to allow full reproducibility. All code used, including Nextflow pipelines and downstream 527 analysis, can be found at https://github.com/alexthiery/otic-reprogramming. Full detailed 528 documentation for the pipeline is also available at https://alexthiery.github.io/otic-reprogramming/. A 529 custom Docker container used for the downstream analysis pipeline can be found at 530 https://hub.docker.com/repository/docker/alexthiery/otic-reprogramming-r analysis. This also allows 531 for interactive exploration of the data.

\section{Bulk-RNA-seq}

534 Bulk-RNA-seq data were processed and aligned to GalGal6 using the default NF-core RNA-seq (v2.0) 535 pipeline ${ }^{54}$ which uses the STAR aligner. Downstream differential expression analysis (Lmx1aE1EGFP vs Sox3U3-EGFP; Sox8OE vs ControlOE) was carried out with the DESeq2 package in $\mathrm{R}^{55}$. 
Hochberg). Differentially expressed transcripts were determined by an absolute log2 fold-change $>1.5$

539 and adjusted $p$-value $<0.05$

\section{Single-cell-RNA-seq alignment}

542 SmartSeq2 single-cell-RNA-seq reads were aligned and processed using a custom Nextflow DSL2 543 pipeline. Adaptor sequences were trimmed using Cutadapt (v2.10). HISAT2 (v2.2.1) was then used 544 to build a genome index from GalGal6 (amended to include a GFP sequence), before extracting splice 545 sites from the GTF and aligning reads. Read counts were obtained using HTSeq (v0.12.4). BAM files 546 from HISAT2 were also passed to Velocyto $(\mathrm{v} 0.17)^{20}$ in order to get spliced and unspliced expression 547 matrices for further downstream analysis.

Single-cell-RNA-seq data analysis

Downstream data analysis was carried out primarily using the Antler $\mathrm{R}$ package (version:

Development2019) ${ }^{18}$. We excluded from the dataset: cells which expressed fewer than $1 \mathrm{k}$ genes or fewer than $500 \mathrm{k}$ reads, cells with more than $6 \%$ reads from mitochondrial genes, genes which were expressed in fewer than 3 cells and genes with CPM $<10$.

Identification of gene modules

556 To identify clusters of genes with correlated expression unbiasedly (gene modules), we used the Antler identifyGeneModules function. Genes which had a Spearman correlation greater than 0.3 with at least 3 other genes were first removed. Genes were then hierarchically clustered iteratively into gene modules and filtered. Gene modules were filtered based on the minimum expression level (5 CPM) and the proportion (0.4) of cells expressing a gene module. The number of final gene modules was set to 40 to achieve reasonably large clusters, which broadly characterise cell type diversity across the dataset. Gene modules were then filtered based on the presence of genes with known expression profiles in ectodermal derivatives, before cells were re-clustered based on the remaining gene modules. We used the expression of known markers to assign cell states. Placodal cells were 
then subset and re-clustered using a new set of gene modules identified from the subset cell population. Three main cell states were identified as otic, epibranchial and OEP populations.

Gene expression dynamics at the otic-epibranchial branching point

To model the transcriptional dynamics at the otic-epibranchial branching point, we ordered cells along pseudotime using Monocle $2^{56}$. The lineage tree was rooted to the earliest cell state (ss8-9) which was comprised mostly of OEPs. The expression of genes along the bifurcation point was then modelled using branch expression analysis modelling (BEAM).

To assess whether individual OEPs simultaneously express markers associated with the otic or epibranchial state, we calculated the co-expression of key otic and epibranchial genes within each of the Monocle branches. First, gene expression was binarized based on the presence or absence of gene expression; then the proportion of cells co-expressing pairs of otic-epibranchial genes within each of the branches was calculated. A Kruskal Wallis test was then used to compare the proportion of cells co-expressing otic and epibranchial genes between the three branches. Post-hoc pairwise comparisons between the OEP branch and the otic or epibranchial branch were carried out using twotailed Wilcoxon rank sum tests.

RNA velocity provides an alternative to Monocle2 for inferring future cell state of individual

cells. Spliced, un-spliced and spanning matrices obtained from Velocyto ${ }^{20}$ were subset in $\mathrm{R}$ based on

genes and cells used to generate the Monocle trajectory. RNA velocity was then calculated using the

Velocyto.R package, where spanning reads were used to fit gene offsets. Single cell velocities as well

\section{ChIP-seq and ATAC-seq alignment and peak calling}

ChIP-seq and ATAC-seq data were processed and aligned to GalGal6 using the default NF-core the Burrows-Wheeler aligner. MACS v2.2.7.1 was used to call broad peaks (FDR < 0.1 ) and narrow peaks (FDR < 0.05) for ChIP-seq and ATAC-seq data, respectively. 


\section{Enhancer discovery}

594 Putative enhancers were identified using a custom Nextflow DSL2 pipeline. First, bedtools (v2.29.2) 595 was used to subset ATAC-seq narrow peaks, which overlap with a broad H3K27ac peak, whilst 596 removing those overlapping with broad H3K27me3 peaks. Remaining ATAC-seq peaks were then 597 annotated with Homer (v4.11), using a GalGal6 GTF which was filtered for protein coding genes. 598 Promoter and exonic peaks were then removed. Motif enrichment and functional enrichment analysis 599 were carried out using Homer and g:Profiler, respectively.

\section{Data availability}

All data have been deposited in Gene Expression Omnibus (GEO; Accession number GSE168089).

Nextflow pipelines and downstream analysis, can be found at https://github.com/alexthiery/otic-

reprogramming. Full detailed documentation is available at https://alexthiery.github.io/otic-

Docker

container

used

can be found at

https://hub.docker.com/repository/docker/alexthiery/otic-reprogramming-r analysis.

\section{References}

Hamburger, V. \& Hamilton, H. L. A series of normal stages in the development of the chick embryo. J Morpho/ 88, 49-92 (1951). 465, doi:10.1016/j.ymeth.2013.10.009 (2014). whole-embryo culture using a filter paper carrier. Dev Dyn 220, 284-289, doi:10.1002/10970177(20010301)220:3<284::AID-DVDY1102>3.0.CO;2-5 (2001).

4 Nagai, H., Lin, M. C. \& Sheng, G. A modified cornish pasty method for ex ovo culture of the chick embryo. Genesis 49, 46-52, doi:10.1002/dvg.20690 (2011). 
50

Streit, A. \& Stern, C. D. Combined whole-mount in situ hybridization and immunohistochemistry in avian embryos. Methods 23, 339-344, doi:10.1006/meth.2000.1146 (2001).

Stern, C. D. \& Holland, P. W. H. Essential Developmental Biology: A Practical Approach., (1993).

Livak, K. J. \& Schmittgen, T. D. Analysis of relative gene expression data using real-time quantitative PCR and the 2(-Delta Delta C(T)) Method. Methods 25, 402-408, doi:10.1006/meth.2001.1262 (2001).

51 Picelli, S. et al. Full-length RNA-seq from single cells using Smart-seq2. Nat Protoc 9, 171181, doi:10.1038/nprot.2014.006 (2014).

Buenrostro, J. D., Giresi, P. G., Zaba, L. C., Chang, H. Y. \& Greenleaf, W. J. Transposition of native chromatin for fast and sensitive epigenomic profiling of open chromatin, DNAbinding proteins and nucleosome position. Nat Methods 10, 1213-1218, doi:10.1038/nmeth.2688 (2013).

Adli, M. \& Bernstein, B. E. Whole-genome chromatin profiling from limited numbers of cells using nano-ChIP-seq. Nat Protoc 6, 1656-1668, doi:10.1038/nprot.2011.402 (2011).

Ewels, P. A. et al. The nf-core framework for community-curated bioinformatics pipelines. Nat Biotechnol 38, 276-278, doi:10.1038/s41587-020-0439-x (2020). Love, M. I., Huber, W. \& Anders, S. Moderated estimation of fold change and dispersion for RNA-seq data with DESeq2. Genome Biol 15, 550, doi:10.1186/s13059-014-0550-8 (2014). Qiu, X. et al. Reversed graph embedding resolves complex single-cell trajectories. Nat Methods 14, 979-982, doi:10.1038/nmeth.4402 (2017).

Acknowledgements. We thank Tatjana Sauka-Spengler and Ruth Williams for assistance with establishing ChIPseq and scRNAseq protocols, Monica Tambalo for help with tissue collection, Rosalinda Guerra and Ewa Kolano-Merlin for technical assistance, and James Briscoe for advice on single cell transcriptomics. Thanks to Claudio D. Stern for comments on the manuscript and the 
646 Streit group for discussions. This work was funded by grants to AS from the Biotechnology and

647 Biological Sciences Research Council (BB/S005536/1; BB/M006964/1) and the National Institute on 648 Deafness and Other Communication Disorders (DC011577).

650 Author contributions. A.L.B., J.C. and A.S. designed experiments; A.L.B. performed RNAseq for 651 Lmx1a-E1-EGFP, Sox3U3-EGFP, Sox8-mCherry/Lmx1aE1-EGFP, mCherry/EGFP populations, 652 scRNAseq, HCR analysis, Sox8/Pax2/Lmx1a OE and some Sox8 OE experiments; J.C. performed 653 ATACseq, ChIPseq, enhancer cloning and testing, in situ hybridisation, qPCR, knock-down and 654 Sox8 OE experiments; J.C. performed the initial analysis of ATACseq and ChIPseq data. A.L.B. 655 interpreted and supervised bioinformatics analysis; J.D. performed some scRNAseq analysis, A.T. 656 analysed all transcriptomic and epigenomic data and established the integrated pipeline; A.L.B. 657 generated all figures; A.L.B., A.T. and A.S. wrote the manuscript. 658

659 Competing interests. The authors declare no competing interests.

Additional information

662 Supplementary information is available for this paper.

663 Correspondence and requests for material should be addressed to Andrea Streit: 